\title{
Oral vasopressin receptor antagonist tolvaptan in right heart failure due to pulmonary hypertension
}

\author{
To the Editor:
}

During the past decade, the prognosis of patients with pulmonary hypertension $(\mathrm{PH})$ has improved due to the development of pulmonary arterial vasodilators [1]. Yet, treatment for right-sided heart failure (RHF) associated with $\mathrm{PH}$ has not improved, despite being the most important prognostic factor for chronic RHF $[2,3]$. Existing treatment strategies for RHF associated with $\mathrm{PH}$ are restricted to administration of loop diuretics and inotropes [4]; however, administration of high doses of loop diuretics may worsen the outcome of chronic heart failure $[5,6]$. In recent years, tolvaptan, an oral, nonpeptide, selective vasopressin V2-receptor antagonist, has been developed for the treatment of hyponatraemia [7], autosomal dominant polycystic kidney disease [8] and heart failure. Used in addition to standard therapy that includes diuretics, tolvaptan improves many signs and symptoms of heart failure without serious adverse events [9]. However, there is no report describing the effect of tolvaptan on RHF, independent of left-sided heart disease.

We evaluated both the short- and long-term effects of tolvaptan, and explored the possibility of loop diuretics dose reduction and improved fluid retention in patients with refractory and loop diuretics-dependent RHF associated with $\mathrm{PH}$.

We enrolled 10 outpatients with chronic RHF associated with $\mathrm{PH}$, who were refractory to loop diuretics, between September 2012 and March 2014 at Keio University Hospital (Tokyo, Japan). This study was an open-label, single-arm, prospective study to investigate the safety and efficacy of tolvaptan in patients with RHF due to $\mathrm{PH}$. The study consisted of a 12-week open-label treatment period with tolvaptan (with visits at baseline, days 1-7, and weeks 2, 4, 6 and 12), and followed the ethical standards of the responsible committee on human experimentation (Keio University School of Medicine ethical committee, Tokyo, Japan) and the Helsinki Declaration. The trial was registered at UMIN before initiating recruitment (identifier number: UMIN000010196). Written informed consent was obtained from all patients in the study. All patients had been previously diagnosed as having idiopathic pulmonary arterial hypertension (IPAH) $(n=5)$ or chronic thromboembolic pulmonary hypertension (CTEPH) $(n=5)$, as confirmed by right heart catheterisation. The baseline dose of furosemide was $60 \mathrm{mg} \cdot \mathrm{day}^{-1}$ for more than 4 weeks and patients were also taking specific therapies for $\mathrm{PH}$ such as oral phosphodiesterase type-5 inhibitors and/or endothelin receptor antagonists. All patients were free from defects such as a predominant left-to-right shunt or any other kind of cardiomyopathy (e.g. ischaemic cardiomyopathy or valvular heart disease). The mean \pm SD age of the patients was $47.8 \pm 15.6$ years and the number of females was five $(50 \%)$. Each patient had been receiving loop diuretics, with an average furosemide dose of $88 \pm 17 \mathrm{mg} \cdot \mathrm{day}^{-1}$. None of the patients had taken another type of diuretic such as spironolactone or hydrochlorothiazide.

The primary end-point was a changed furosemide dosage at week 12 relative to baseline. Tolvaptan was administered orally at a dose of $3.75 \mathrm{mg}, 7.5 \mathrm{mg}$ or $15 \mathrm{mg}$ per day. For safety reasons, all participants commenced tolvaptan at a dose of $3.75 \mathrm{mg} \cdot$ day $^{-1}$ for 3 days. Provided urine output did not increase during this initial 3-day period, the dosage of tolvaptan was increased to $7.5 \mathrm{mg} \cdot \mathrm{day}^{-1}$ and $15 \mathrm{mg} \cdot \mathrm{day}^{-1}$. The dosage of furosemide was reduced in cases satisfying the following conditions: 1) urine output was greater than baseline levels, and more than $1000 \mathrm{~mL}$ per day; and 2) there was no evidence of increased body weight compared with the previous day. In cases free from increased fluid retention (leg oedema, hepatomegaly or jugular vein distention), a reduction of furosemide would be considered from $160 \mathrm{mg}$, $120 \mathrm{mg}, 80 \mathrm{mg}, 60 \mathrm{mg}, 40 \mathrm{mg}, 20 \mathrm{mg}$, to $10 \mathrm{mg}$ per day.

The mean \pm SD dosage of tolvaptan at day 7 and week 12 were $6.38 \pm 1.8 \mathrm{mg} \cdot \mathrm{day}^{-1}$ and $7.87 \pm 4.1 \mathrm{mg} \cdot \mathrm{day}^{-1}$, respectively. During the adjustment of tolvaptan dosing, neither overcorrection of serum sodium $\left(>150 \mathrm{mEq} \cdot \mathrm{L}^{-1}\right)$ or elevated liver enzymes were noted, and the main adverse event was a dry mouth $(80 \%)$. There were no withdrawals from the study due to adverse events. There were no patients who developed hypotension or experienced worsening heart failure during treatment.

The comparisons of clinical variables at baseline and post-tolvaptan treatment are shown in figure 1. The mean dose of furosemide administered at day 7 and week 12 compared with baseline was significantly reduced by tolvaptan treatment $(p=0.001)$. Tolvaptan was also associated with a significant decrease in average urine osmolality and brain natriuretic peptide levels at week 12 compared with baseline $(p=0.001$ 
and $\mathrm{p}=0.014$, respectively). Improvements in physical assessments (body weight, leg oedema and jugular vein distention) were also associated with tolvaptan treatment. Serum sodium, potassium and creatinine concentrations showed no significant changes during the time course of the treatment. Blood pressure and heart rate remained unchanged during treatment. The effect of the study drugs on self-assessed health
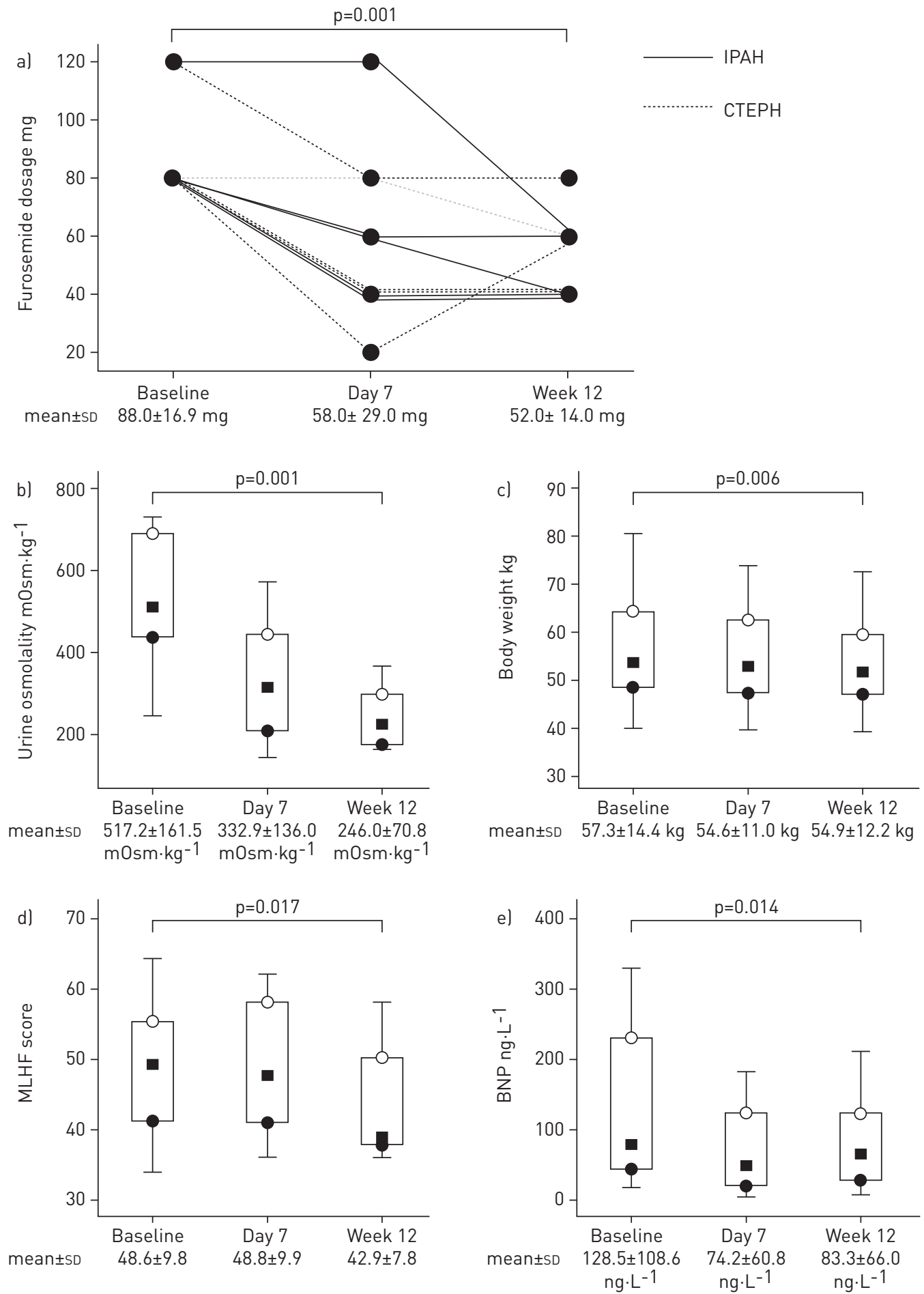

FIGURE 1 a) Line graphs of furosemide dosage in each patient during tolvaptan treatment. Box and whisker plots of b) urine osmolality, c) body weight, d) Minnesota Living with Heart Failure Questionnaire (MLHF) score, and e) brain natriuretic peptide (BNP) at baseline, day 7 and week 12 after commencement of tolvaptan treatment. Squares represent the mean, circles represent the 25th and 75th percentiles, and whiskers represent the range. IPAH: idiopathic pulmonary arterial hypertension; CTEPH: chronic thromboembolic pulmonary hypertension. 
status was determined using the Minnesota Living with Heart Failure Questionnaire (MLHF), and the average score was significantly improved $(\mathrm{p}=0.017)$.

All data are expressed as mean \pm SD. Patients who took at least one dose of tolvaptan were included in the set for analyses. A comparison of data obtained at baseline, day 7 and week 12 was made using the nonparametric Friedman's test. The significance level in all statistical analyses was set at 5\% (two-sided). All analyses were performed using SPSS statistical software (version 20.0; IBM Corp., Armonk, NY, USA).

From these results, tolvaptan treatment achieved a significant reduction in furosemide dosage over a 12-week period in patients with chronic RHF associated with $\mathrm{PH}$, and did so without any serious adverse events. In addition, tolvaptan administration also produced significantly improved brain natriuretic peptide levels and physical findings of fluid retention associated with a decrease in urine osmolality.

While RHF is an established risk factor for a poor prognosis in patients with $\mathrm{PH}$, there is no established treatment for targeting RHF itself. Digoxin was tested in a small study of 17 patients with pulmonary arterial hypertension (PAH), which suggested digoxin has acute haemodynamic effects [10]. In a basic study, Nagendran et al. [11] demonstrated that sildenafil may have direct inotropic effects. Use of high-dose loop diuretics is unavoidable in the treatment of fluid retention in classical RHF associated with $\mathrm{PH}$. However, use of these diuretics is accompanied by a poor prognosis and worsening renal failure due to upregulation of the renin-angiotensin-aldosterone system and sympathetic nerve activation in patients with chronic heart failure [9]. In such cases, the vasopressin type 2-receptor selective antagonist, tolvaptan, is considered a potent therapy, because the upregulation of vasopressin is associated with the aggravation of the renin-angiotensin-aldosterone system [12]. Excess vasopressin produces fluid retention via a direct effect on the renal collecting duct in patients with chronic heart failure. As a result of a continuous vasopressin excess, circulating blood volume and urine osmolality will increase, even if using high-dose loop diuretics. This phenomenon is known as loop diuretics resistance [13].

We expect tolvaptan has the potential to become the first potent drug leading to long-term survival benefits in patients with RHF due to $\mathrm{PH}$, for whom there is currently no established treatment. This study is prospective, and is a small single-armed pilot study lacking long-term observations. However, its positive results suggest that there should be no hesitation to pursue further investigation in a multicentre randomised control study and confirm the beneficial effects of tolvaptan in this type of patient. improvements in PH http://ow.ly/Lopej

Yuichi Tamura ${ }^{1,2,3,4}$, Mai Kimura ${ }^{1}$, Makoto Takei ${ }^{1}$, Tomohiko Ono ${ }^{1}$, Masataka Kuwana ${ }^{5}$, Toru Satoh ${ }^{6}$, Keiichi Fukuda ${ }^{1}$ and Marc Humbert ${ }^{2,3,4}$

${ }^{1}$ Dept of Cardiology, Keio University School of Medicine, Tokyo, Japan. ${ }^{2}$ Faculté de Médecine, Université Paris-Sud, Le Kremlin Bicêtre, France. ${ }^{3}$ Service de Pneumologie, DHU Thorax Innovation, Hôpital Bicêtre, Assistance Publique Hôpitaux de Paris, Le Kremlin-Bicêtre, France. ${ }^{4}$ Inserm UMR_S 999, LabExLERMIT, Centre Chirurgical Marie Lannelongue, Le Plessis Robinson, France. ${ }^{5}$ Dept of Allergy and Rheumatology, Nippon Medical School, Tokyo, Japan. ${ }^{6}$ Dept of Cardiology, Kyorin University School of Medicine, Tokyo, Japan.

Correspondence: Yuichi Tamura, Dept of Cardiology, Keio University School of Medicine, 35 Shinanomachi Shinjuku-ku, Tokyo 160-8582, Japan. E-mail: u1@ta-mu.net

Received: Dec 252014 | Accepted after revision: March 302015 | First published online: April 302015

Clinical trial: This study is registered at www.umin.ac.jp/ctr/ with identifier number UMIN000010196.

Support statement: This work was supported by Health and Labor Sciences Research Grants in Japan.

Conflict of interest: Disclosures can be found alongside the online version of this article at erj.ersjournals.com

Acknowledgements: The authors acknowledge the medical staff of the Dept of Cardiology, Keio University Hospital, Tokyo, Japan, for completion of medical records.

\section{References}

1 Humbert M, Sitbon O, Chaouat A, et al. Survival in patients with idiopathic, familial, and anorexigen-associated pulmonary arterial hypertension in the modern management era. Circulation 2010; 122: 156-163.

2 Galiè N, Hoeper MM, Humbert M, et al. Guidelines for the diagnosis and treatment of pulmonary hypertension: the Task Force for the Diagnosis and Treatment of Pulmonary Hypertension of the European Society of Cardiology (ESC) and the European Respiratory Society (ERS), endorsed by the International Society of Heart and Lung Transplantation (ISHLT). Eur Heart J 2009; 30: 2493-2537.

3 Vonk-Noordegraaf A, Westerhof N. Describing right ventricular function. Eur Respir J 2013; 41: 1419-1423.

4 Vonk-Noordegraaf A, Haddad F, Chin KM, et al. Right heart adaptation to pulmonary arterial hypertension: physiology and pathobiology. J Am Coll Cardiol 2013; 62: Suppl., D22-D33. 
5 Neuberg GW, Miller AB, O'Connor CM, et al. Diuretic resistance predicts mortality in patients with advanced heart failure. Am Heart J 2002; 144: 31-38.

6 Mielniczuk LM, Tsang SW, Desai AS, et al. The association between high-dose diuretics and clinical stability in ambulatory chronic heart failure patients. J Card Fail 2008; 14: 388-393.

7 Schrier RW, Gross P, Gheorghiade M, et al. Tolvaptan, a selective oral vasopressin V2-receptor antagonist, for hyponatremia. N Engl J Med 2006; 355: 2099-2112.

8 Torres VE, Chapman AB, Devuyst O, et al. Tolvaptan in patients with autosomal dominant polycystic kidney disease. N Engl J Med 2012; 367: 2407-2418.

9 Gheorghiade M, Konstam MA, Burnett JCJr, et al. Short-term clinical effects of tolvaptan, an oral vasopressin antagonist, in patients hospitalized for heart failure: the EVEREST Clinical Status Trials. JAMA 2007; 297: $1332-1343$.

10 Boldt J, Wollbrück M, Kuhn D, et al. Do plasma levels of circulating soluble adhesion molecules differ between surviving and nonsurviving critically ill patients? Chest 1995; 107: 787-792.

11 Nagendran J, Archer SL, Soliman D, et al. Phosphodiesterase type 5 is highly expressed in the hypertrophied human right ventricle, and acute inhibition of phosphodiesterase type 5 improves contractility. Circulation 2007; 116: $238-248$

12 McKinley MJ, Mathai ML, McAllen RM, et al. Vasopressin secretion: osmotic and hormonal regulation by the lamina terminalis. J Neuroendocrinol 2004; 16: 340-347.

13 Gupta S, Neyses L. Diuretic usage in heart failure: a continuing conundrum in 2005. Eur Heart J 2005; 26: 644-649.

Eur Respir J 2015; 46: 283-286 ｜ＤOI: 10.1183/09031936.00044915 | Copyright @ERS 2015 